\title{
Le dilemme inévitable de l'action humanitaire
}

The humanitarian dilemma - an eternal phenomenon of humanitarian action

\author{
Michael Schloms
}

\section{(2) OpenEdition}

1 Journals

\section{Édition électronique}

URL : https://journals.openedition.org/conflits/1924

DOI : 10.4000/conflits. 1924

ISSN : $1777-5345$

Éditeur :

CECLS - Centre d'études sur les conflits - Liberté et sécurité, L'Harmattan

\section{Édition imprimée}

Date de publication : 1 décembre 2005

Pagination : 85-102

ISBN : 2-296-00230-7

ISSN : 1157-996X

Référence électronique

Michael Schloms, «Le dilemme inévitable de l'action humanitaire », Cultures \& Conflits [En ligne], 60 | hiver 2005, mis en ligne le 15 janvier 2006, consulté le 27 septembre 2021. URL : http:// journals.openedition.org/conflits/1924; DOI : https://doi.org/10.4000/conflits.1924

Ce document a été généré automatiquement le 27 septembre 2021.

Creative Commons License 


\title{
Le dilemme inévitable de l'action humanitaire
}

The humanitarian dilemma - an eternal phenomenon of humanitarian action

\author{
Michael Schloms
}

1 Depuis le début des années 1990, l'aide humanitaire internationale est au centre d'un débat public et académique. Des missions humanitaires, comme en Afghanistan sous le régime des Talibans, dans les camps de réfugiés rwandais ou dans la guerre civile soudanaise ont mis en question les motifs et les principes du mouvement humanitaire. Selon Tony Vaux de l'organisation britannique Oxfam, la Guerre Froide représentait « l'âge d'or » du mouvement humanitaire, car l'espace de manœuvre des humanitaires

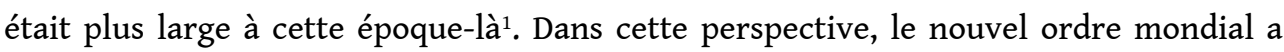
créé un environnement dans lequel le mandat humanitaire est devenu une tâche difficile, ambiguë et beaucoup plus politique qu'auparavant. Dans un premier temps, l'article analysera le débat actuel sur la crise humanitaire. Puis, nous élaborerons les caractéristiques de l'ambiguïé de l'action humanitaire et les raisons de son dilemme. Enfin, l'article présentera les différentes stratégies des organisations humanitaires qui se trouvent dans une situation dans laquelle leurs obligations éthiques sont mises en cause.

Compte tenu des raisons structurelles du dilemme qui accompagne l'histoire humanitaire depuis son début, les revendications actuelles pour un «nouvel humanitarisme" se relativisent. En même temps, étant donné l'hétérogénéité des organisations humanitaires et leur relation à leurs principes fondamentaux, il est évident que l'action humanitaire se trouve dans une crise de cohérence. 


\section{Le débat : une nouvelle action humanitaire dans un nouvel ordre mondial}

\section{Le constat}

3 Selon un nombre considérable d'auteurs, l'humanitarisme d'aujourd'hui se trouve discrédité à cause des changements fondamentaux de l'après-Guerre Froide. Fox conclut que le système de l'aide internationale " a évidemment été discrédité et a perdu sa confiance en soi $»^{2}$. L'argument principal est le suivant: du fait de l'effondrement du contrôle gouvernemental, et des infrastructures publiques en tant que cause et conséquence des conflits armés, l'action humanitaire se déroule dans un espace sans règles morales ou juridiques. Par conséquent, les organisations ont du mal à faire respecter les principes de leur action qui leur permettraient d'atteindre les populations vulnérables dans une crise humanitaire. De plus, les agences humanitaires font face au danger de la manipulation ou de la diversion de leur aide. Lindenberg et Bryant résument que «les plus grands dilemmes pour des ONG qui travaillent dans les formes d'urgences les plus complexes portent sur le fait de savoir si les actions qui sauvent des vies participent à la perpétuation du conflit » ${ }^{3}$. Les auteurs mentionnent comme "dilemmes » de l'action humanitaire l'assistance indirecte à la déportation des populations, la diversion de l'aide, la perpétuation d'une économie de guerre, l'escalade du conflit et de la compétition entre plusieurs groupes (en assistant des réfugiés mais pas des résidents, par exemple), et l'affaiblissement des capacités locales nécessaires pour faire face à une crise ${ }^{4}$. Du fait des changements dans le monde de l'après-Guerre Froide, ces dilemmes apparaissent plus souvent qu'auparavant. La crise rwandaise, notamment, était une expérience extrêmement traumatique pour tout le système d'aide internationale. Nicholas Leader, quant à lui, conclut que "les voix uniques des années soixante-dix et quatre-vingt qui ont reproché aux agences [humanitaires] d'attiser des conflits [et] de nourrir des bourreaux (...) sont devenues un chœur dans les années quatre-vingt-dix ${ }^{5}$. Dans cette perspective, ces reproches ont eu comme conséquence la «crise de confiance $»^{6}$ du mouvement humanitaire.

4 Au-delà de l'hypothèse de la fin de la Guerre Froide comme raison principale à la crise de l'humanitaire, un deuxième aspect domine le débat sur l'aide discréditée : les droits de l'Homme. Selon plusieurs auteurs, la protection des droits de l'Homme est devenue une tâche humanitaire et fait partie du mandat de certaines organisations. Médecins Sans Frontières (MSF) est souvent mentionnée comme prototype d'une telle organisation. En effet, dans son discours à Oslo lors de l'attribution du Prix Nobel en 1999, le président de MSF International déclare: "Plus que l'assistance matérielle, nous essayons de permettre aux individus de regagner leurs droits et leur dignité en tant qu'être humain $»^{7}$. Rony Brauman, dans un entretien sur le dilemme humanitaire, présente une conception similaire de l'action humanitaire: "Au-delà du soin, l'action humanitaire permet de pointer le doigt vers l'injustice, de désigner l'inacceptable $»^{8}$.

5 Un dilemme apparaît au moment où une organisation fait face au choix entre l'obligation morale de sauver des vies, et l'obligation de ne pas soutenir un régime responsable de violations massives et systématiques des droits de l'Homme. Généralement, l'obligation humanitaire d'assister des personnes qui en ont besoin et l'obligation de protéger et promouvoir les droits de l'Homme sont vues comme des 
obligations différentes, comme des objectifs de même valeur qui ainsi, dans certaines circonstances, s'excluent l'un l'autre.

6 Ainsi, le débat sur l'intégrité morale de l'action humanitaire d'aujourd'hui constate que le système se trouve dans une crise fondamentale. Cette crise marque la fin de l'âge d'or de l'humanitarisme et met en question les obligations et les convictions fondamentales de la tradition humanitaire.

\section{Les conclusions}

7 Le débat sur le dilemme humanitaire met en cause les principes classiques de l'action humanitaire. L'argument est de dire qu'une crise fondamentale nécessite des contremesures également fondamentales. Fox soutient qu'il faut moderniser l'action humanitaire pour qu'elle puisse faire face aux défis modernes :

« Il y a un 'nouvel humanitarisme' pour le nouveau millénaire. Il est fondé sur des principes et les droits de l'Homme, il est sensible au politique et adapté pour renforcer la paix et la stabilité dans le monde en voie de développement. Il offre aux agences humanitaires un nouvel étendard moral à suivre $»^{9}$.

Pour l'essentiel, ce «nouvel humanitarisme » réunit deux approches qui visent à éviter les situations de dilemme et d'ambiguité : le pragmatisme et la politisation.

Le pragmatisme cherche à améliorer la qualité du travail humanitaire sur le terrain. Un élément important est la codification de plusieurs principes d'action dans des protocoles, dans les codes de conduite, etc. Généralement, ces textes codifient certains standards d'action par rapport aux objectifs et l'organisation de la mission (contrôle de la distribution de l'aide, distribution selon les besoins etc.). Un type de codes de conduite concerne l'action humanitaire en général (Red Cross-Red Crescent / NGO Code of Conduct, SPHERE project), une autre catégorie définit des principes d'action dans des missions singulières, notamment dans les cas où le travail humanitaire est controversé $^{10}$. L'objectif de la codification des principes est "d'inspirer l'action humanitaire (...) et de l'orienter de manière à ce qu'elle ne soit pas pervertie de sa mission humanitaire. En bref, ce sont des règles pour dîner avec le diable sans être mangé ou corrompu $»^{11}$.

Le deuxième élément de la stratégie pragmatique est la coordination qui « $a$ une réputation bien établie d'être l'un des domaines les plus problématiques de l'action humanitaire $\aleph^{12}$. La diversité des organisations et des donateurs qui sont engagés dans une urgence humanitaire est souvent vue comme une faiblesse structurelle de l'action humanitaire, surtout dans les cas où les objectifs humanitaires sont mis en cause. Une autre faiblesse structurelle concerne la capacité des organisations humanitaires à analyser le contexte de leur travail et à évaluer leurs propres expériences. L'intérêt des donateurs à minimiser les coûts administratifs d'une agence et les contrats à court terme du personnel humanitaire expliquent pourquoi bien des auteurs constatent que les organisations humanitaires ont tendance à réinventer la roue ${ }^{13}$. L'adoption d'une approche commune, l'établissement d'un mécanisme élaboré de coordination et l'échange permanent d'informations et d'analyses font donc partie de l'approche pragmatique.

11 La deuxième approche qui constitue le "nouvel humanitarisme" demande une orientation plus politique des organisations humanitaires. L'argument principal consiste à dire que l'action humanitaire a besoin d'un poids politique pour pouvoir 
puisse imposer le mandat humanitaire ${ }^{14}$. Ce modèle correspond à l'initiative de quelques ONG françaises qui cherchaient à établir un « droit d'ingérence ».

Une telle "politisation de la philosophie humanitaire ${ }^{15}$ est demandée par des auteurs qui critiquent l'action humanitaire traditionnelle pour sa naïveté, sa philanthropie et son caractère purement émotionnel. La politisation de l'humanitarisme correspondrait à une rupture avec une telle tradition, «ce serait quelque chose comme une révolution copernicienne dans la pensée philanthropique ${ }^{16}$. De plus, une telle politisation ne concerne pas seulement la philosophie et les moyens des agences humanitaires, mais aussi leur objectif. Le renforcement de la paix est souvent regardé comme une tâche humanitaire qui permettrait de restituer l'intégrité morale du mouvement humanitaire. Au lieu de sauver des vies avec des moyens médicaux, l'aide devrait, au premier lieu, renforcer « les capacités de la paix " pour ne pas « causer de dommage $»^{17}$. Ainsi, l'approche "do no harm» est devenue un nouveau paradigme de l'aide internationale y compris de l'action humanitaire.

\section{Ambiguïté structurelle et dilemme concret}

13 L'action humanitaire d'aujourd'hui doit-elle faire face aux nouveaux défis qui nécessitent une adaptation fondamentale de l'humanitarisme? Nous traiterons cette question en nous appuyant sur le débat humanitaire. Nous présenterons d'abord une terminologie qui distingue l'ambiguïté structurelle du dilemme concret. Cela nous permettra de mettre en cause l'hypothèse dominante selon laquelle le dilemme humanitaire découle du conflit moral entre l'humanitarisme d'une part, et la protection des droits de l'Homme et de la paix, d'autre part.

\section{La terminologie : ambiguïté et dilemme}

Dans son analyse de l'histoire de l'humanitarisme, Alain Destexhe, ancien secrétaire général de MSF International, constate que "s'il fallait caractériser d'un seul mot l'action humanitaire, ce serait par 'ambiguïté' ${ }^{18}$. En fait, l'ambiguïté fait partie du travail humanitaire dans la mesure où les organisations humanitaires n'ont pas les moyens pour déterminer les conditions de leur travail. En revanche, pour leur liberté d'action et le respect des principes de leur travail (" espace humanitaire»), les organisations sont dépendantes des décisions et des intérêts des acteurs politiques. En d'autres termes, les agences humanitaires ne peuvent pas déterminer d'une manière indépendante les conditions et donc, les effets de leur action. Elles agissent dans un cadre défini notamment par les intérêts des responsables politiques du pays ou de la région qui se trouve en crise. Aussi, les institutions politiques dans les pays donateurs qui financent les activités humanitaires ont des moyens pour influencer le travail humanitaire. En un mot, l'ambiguité est un élément structurel de l'action humanitaire qui fait partie de l'histoire de l'humanitarisme moderne.

15 Le terme de "dilemme ", en revanche, décrit une situation concrète, un conflit éthique dans un cas donné. Alors que l'ambiguïté est une caractéristique générale de l'action humanitaire, le dilemme humanitaire dépend de certaines conditions. Selon une définition générale, un dilemme est une situation dans laquelle un acteur se trouve face à au moins deux obligations morales qui, premièrement, sont de même valeur et qui, deuxièmement, s'excluent l'une l'autre ${ }^{19}$. Il faut insister sur le fait qu'un dilemme est 
plus qu'une décision difficile ou douloureuse, dans la mesure où un dilemme ne permet pas de choisir entre des obligations de valeurs différentes ${ }^{20}$. Dans une mission humanitaire, le choix entre plusieurs groupes de population (enfants, réfugiés, etc.), par exemple, est une décision difficile parce qu'on exclut des groupes de l'aide humanitaire alors qu'ils en auraient besoin. Mais il ne s'agit pas d'un dilemme tant que les besoins d'un certain groupe sont plus grands que ceux des autres. Dans ce cas-là, une organisation humanitaire aura l'obligation morale d'aider les plus vulnérables. Dans la perspective de l'humanitarisme traditionnel, l'objectif d'améliorer le niveau de vie de toute une population n'a pas la même valeur morale.

Quelles sont donc les obligations morales qui sont capables de déclencher un dilemme humanitaire? Selon le débat susmentionné, la protection des droits de l'Homme, l'engagement pour la paix, pour la stabilité et pour la démocratie sont des obligations principales qui déterminent l'action humanitaire et qui sont responsables des conflits éthiques de l'après-Guerre Froide. En d'autres termes, les objectifs de l'humanitarisme traditionnel (assistance médicale d'urgence selon les principes comme l'humanité, l'impartialité et la neutralité) rivalisent avec les objectifs qui résultent du nouvel ordre mondial (droits de l'Homme, paix, etc.).

D'abord, il est nécessaire d'analyser le cadre moral de l'action humanitaire «traditionnelle ». En général, l'action humanitaire fait face à trois groupes d'acteurs qui influencent les obligations morales d'un acteur humanitaire. Premièrement, la population vulnérable, c'est-à-dire l'existence de souffrance humaine, détermine le comportement d'une organisation humanitaire. La volonté de soulager la souffrance est l'obligation initiale du mouvement, et reste donc au cœur de l'éthique humanitaire (« impératif humanitaire »).

Deuxièmement, c'est le cadre éthique de l'organisation elle-même qui influence son comportement sur le terrain. Ce cadre éthique se compose du mandat de l'activité spécifique de l'organisation (aide médicale, nutrition, agriculture etc.), de son histoire de fondation et sa tradition. Nous allons voir que le mouvement humanitaire s'est diversifié au cours des dernières décennies et que cette diversification met en question les principes de l'humanitarisme de la "lignée genevoise ${ }^{21}$.

19 La troisième influence dérive des intérêts politiques d'acteurs externes (au pays bénéficiaire et au pays donateur de l'aide). L'environnement politique détermine donc des conditions à respecter par les organisations humanitaires, conditions qui ont aussi une connotation morale. Les effets du travail humanitaire sur une économie de guerre ou sur la stabilité d'un régime antidémocratique, par exemple, peuvent poser des problèmes éthiques pour une agence humanitaire. 
Fig. 1 : Les obligations morales de l'action humanitaire

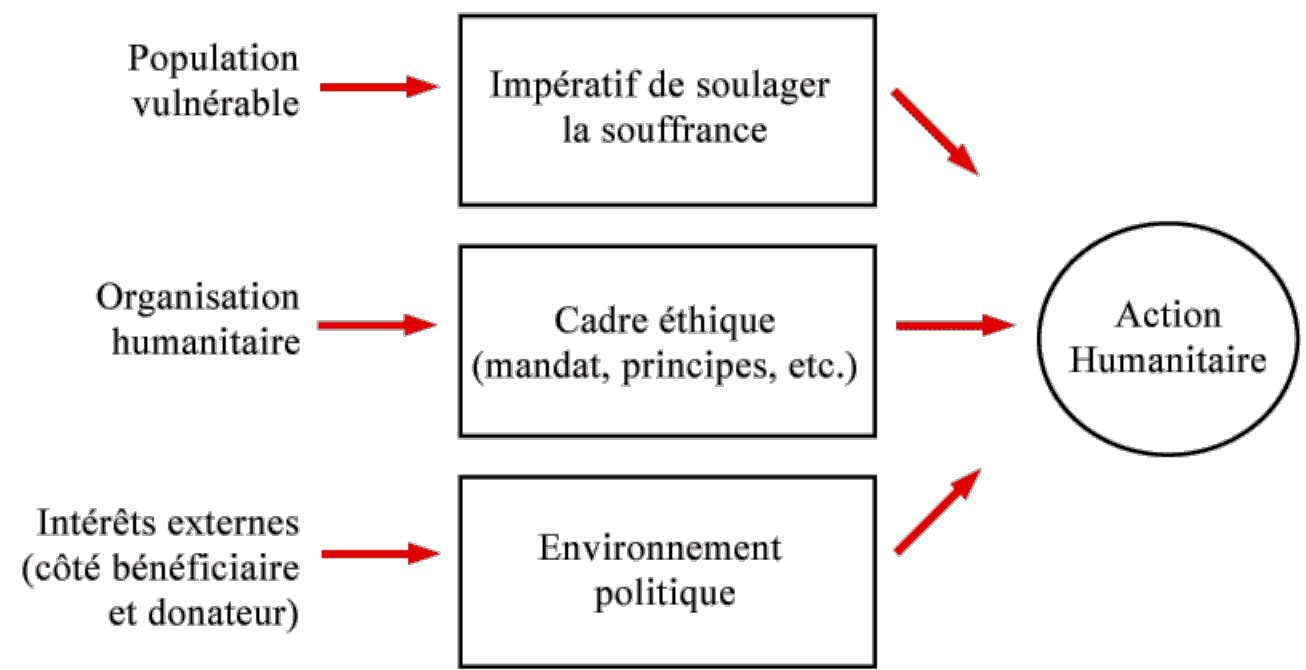

Le schéma nous permet de mieux comprendre le comportement d'un acteur humanitaire dans une situation de conflit moral. Une organisation peut accorder un poids différent aux trois sources d'obligations. Cela permet de comprendre les différentes stratégies des agences d'aide. Pour cela, il est d'abord nécessaire de déterminer les facteurs qui déclenchent un dilemme moral et de définir le rôle que joue la protection des droits de l'Homme et de la paix.

\section{Les sources du dilemme humanitaire}

21 Pour identifier les facteurs qui déclenchent un dilemme moral dans l'action humanitaire, il est nécessaire d'analyser le comportement des acteurs humanitaires dans des cas concrets. Parmi les cas les plus ambigus dans l'histoire de l'humanitaire, figurentles crises de réfugiés et les famines ${ }^{22}$. Dès qu'une crise humanitaire est liée à des facteurs politiques ou déclenchée par ceux-ci, il est probable que les acteurs humanitaires feront face à des obligations divergentes. Selon les obligations morales de l'humanitarisme présentées ci-dessus, il faut choisir un cas qui remplit trois conditions pour analyser le dilemme humanitaire. D'abord, il faut que la survie d'une population soit en danger pour obliger les acteurs humanitaires à soulager activement la souffrance (impératif humanitaire). Deuxièmement, il faut qu'une multitude d'acteurs soit engagée dans cette mission pour pouvoir analyser des mandats et des traditions différents (cadre éthique organisationnel) au sein du mouvement humanitaire. Troisièmement, pour une analyse du dilemme humanitaire, il est nécessaire qu'une crise touche des intérêts politiques massifs (environnement politique qui réduit l'espace de manœuvre des agences d'aide) : l'assistance aux groupes de populations délivrée par des organisations étrangères touche les intérêts politiques des responsables en place. De plus, si la crise se déroule dans un pays ou une région qui est au cœur de querelles diplomatiques, voire militaires, la mission humanitaire va aussi concerner les intérêts des bailleurs de fonds.

Il y a plusieurs cas dans l'histoire de l'humanitarisme qui répondent à ces conditions, par exemple la famine en Russie (1921-1923), la famine au Biafra (1968-1970), la crise au Cambodge (1979-1980), la famine en Ethiopie (1984-1985) ou les crises de l'après-Guerre Froide au Rwanda, en Somalie ou en Afghanistan sous les Talibans. Un cas qui a 
récemment déclenché des controverses parmi les organisations d'aide et qui illustre bien le dilemme humanitaire, est celui de la Corée du Nord. Les acteurs ont dû faire face à des besoins humanitaires énormes ( 5 à $10 \%$ de la population totale sont morts de famine, soit un à deux millions d'individus ${ }^{23}$ ), qui ont eu comme conséquence l'une des plus longues et plus coûteuses missions humanitaires dans l'histoire des Nations Unies $^{24}$. Des organisations d'aide peu nombreuses mais très diverses se sont engagées et continuent à s'engager dans plusieurs secteurs de l'action humanitaire (aide alimentaire, nutrition, agriculture, santé publique, etc.). Enfin, le travail humanitaire en Corée du Nord est pour les autorités coréennes ainsi que pour les pays donateurs (notamment les Etats-Unis, le Japon, la Corée du Sud et les Etats membres de l'Union européenne) une activité hautement sensible pour des raisons politiques. Prenons donc l'exemple de la Corée du Nord pour élucider le dilemme de l'action humanitaire.

L'ambiguïé structurelle du travail humanitaire est évidente en Corée du Nord dans la mesure où la liberté d'action est très limitée : les agences d'aide ne peuvent pas ellesmêmes identifier les besoins de la population, elles ne peuvent pas définir les institutions ou les individus bénéficiaires indépendamment, et elles ne peuvent pas évaluer les effets de leurs projets sur la santé et le statut nutritionnel de la population ${ }^{25}$. Les restrictions concernant les conditions de travail ont déclenché des réactions très diverses de la part des acteurs humanitaires. Toutes les agences considèrent leur travail en Corée du Nord comme étant une tâche difficile, mais seul un certain groupe d'acteurs se trouve face à un dilemme.

Ce sont les ONG médicales qui sont engagées notamment dans la médecine d'urgence, la nutrition et la santé publique, qui ont fait face à un dilemme et qui, finalement, ont pris la décision de se retirer de la Corée du Nord. Médecins Sans Frontières (MSF) et Médecins du Monde (MDM) en 1998, Action Contre La Faim (ACF) et OXFAM en 2000 ont quitté le pays pour des raisons éthiques ${ }^{26}$. Les organisations médicales ont vu un conflit insoluble entre leur mandat humanitaire et les restrictions en place. Par exemple, à l'époque, le directeur général de MSF, Erich Goemare, conclut: «l'aide humanitaire ne peut aider les plus vulnérables que si elle est librement distribuée ${ }^{27}$. ACF justifie sa décision en soulignant le "manque d'accès aux populations les plus vulnérables $»^{28}$. Aucune ONG qui s'est retirée du pays n'a douté de l'existence d'une famine en Corée du Nord.

Ces organisations font donc référence à l'impossibilité d'aider les plus vulnérables comme raison principale de leur départ du pays. D'autres explications, comme une distribution discriminatoire de l'aide par exemple, jouent aussi un rôle, notamment par rapport aux positions d'ACF et MSF. Selon ACF «les autorités privent délibérément d'assistance des centaines de milliers de Coréens " et l'aide "n'alimente, de fait, que les populations que le régime a d'emblée choisi de privilégier ${ }^{29}$. Il faut ajouter qu'une telle conclusion manque d'analyse profonde et paraît plutôt douteuse ${ }^{30}$.

Selon l'hypothèse susmentionnée, c'est le conflit entre l'engagement pour les droits de l'Homme ou la démocratie d'une part et l'impératif humanitaire d'autre part, qui est à la base du dilemme humanitaire. Pourtant, aucune agence n'a justifié la décision de cesser ses activités en se référant à la situation des droits de l'Homme en soi ou au système totalitaire en place.

Or, il faut mettre en exergue que les références au système politique et à l'oppression des droits de l'Homme qui ne jouent qu'un rôle supplémentaire dans l'argumentation des ONG qui ont quitté la Corée du Nord. La raison principale est évidemment la conviction selon laquelle l'aide humanitaire ne peut pas respecter les principes de 
l'action humanitaire dans ce pays. En effet, le principe de l'humanité (ou l'impératif humanitaire) et le principe de la proportionnalité s'excluent l'un l'autre. Le conflit entre ces deux principes fondamentaux et essentiels de l'action humanitaire est donc à la base du dilemme moral.

Le cas de la Corée du Nord illustre ainsi qu'a priori, les violations des droits de l'Homme ou la présence d'un système totalitaire en soi ne déclenchent pas un dilemme pour les organisations humanitaires. Agir dans un environnement non démocratique fait partie du travail quotidien des agences d'aide. Des missions humanitaires dans des pays démocratiques où les droits de l'Homme sont respectés sont rarissimes. Le travail humanitaire dans les régions en crise est donc, selon notre terminologie, ambigu. Or, pour déclencher un vrai dilemme il faut que le principe de la proportionnalité soit mis en cause. Le non-respect de la démocratie, de la paix ou des droits de l'Homme pose un dilemme humanitaire car il empêche la distribution de l'aide.

D'autres missions humanitaires confirment cette hypothèse. En Afghanistan, par exemple, des ONG comme Oxfam ou MSF ont décidé de ne pas coopérer avec les Talibans à cause de la discrimination faite aux femmes sous leur régime ${ }^{31}$. Cette démarche a crée des dilemmes dans la mesure où les femmes étaient l'un des groupes les plus vulnérables. Donc, l'impossibilité d'obéir à l'impératif humanitaire et, en même temps, d'atteindre les personnes les plus vulnérables était au cœur du débat. Il ne s'agissait pas, à l'époque, d'un conflit moral qui opposait l'éthique humanitaire à une éthique des droits de l'Homme ou de la paix.

Le débat entre différents acteurs humanitaires autour de la décision de quitter un pays en crise illustre que des agences d'aide ne prennent pas en compte la nature du dilemme humanitaire. En revanche, le débat parmi les organisations a les mêmes caractéristiques que le débat public présenté ci-dessus. Ainsi, la critique principale consiste à dire que les organisations qui quittent une mission ne respectent pas leur obligation humanitaire en mélangeant travail humanitaire, engagement politique et protection des droits de l'Homme. Or, pour l'essentiel, c'est l'éthique humanitaire qui est mise en cause. Un débat sur les principes de l'humanitarisme, notamment sur le rôle de la proportionnalité aurait été nécessaire mais n'a pas eu lieu ${ }^{32}$.

Finalement, les raisons du dilemme proviennent de l'éthique humanitaire (le conflit entre humanité et impartialité) et ne sont donc pas le résultat d'un conflit qui oppose l'éthique humanitaire à d'autres valeurs morales (les droits de l'Homme, la paix etc.). Il est donc évident que le débat actuel, qui regarde en premier lieu le rôle des droits de l'Homme et de la paix par rapport à l'action humanitaire, ne fait qu'à peine référence aux raisons du dilemme humanitaire qui, elles, font partie du noyau dur de l'éthique humanitaire. Autrement dit, le débat se trouve détourné par des phénomènes récents alors que le dilemme humanitaire accompagne l'histoire de l'humanitarisme depuis des siècles.

\section{Les acteurs humanitaires et leurs stratégies face au dilemme}

32 Il va de soi qu'un dilemme au sens strict du terme ne peut pas être résolu d'une manière qui éradique tout conflit moral et toute ambivalence. Néanmoins, il existe différentes approches pour faire face à un dilemme humanitaire. Nous allons voir que 
le comportement des organisations d'aide dans des missions qui sont moralement difficiles est très différent. Cela reflète une diversité de cadres éthiques qui, enfin, met en cause la cohérence de l'action humanitaire.

L'humanitarisme, comme mentionné ci-dessus, fait face à trois sources d'obligations morales : l'impératif humanitaire, le cadre éthique de l'organisation et l'environnement politique. Dès lors, une agence peut prendre ses décisions en s'orientant vers un de ces facteurs. Elle peut donc obéir uniquement à l'impératif humanitaire (l'approche affective), elle peut s'orienter vers ses propres principes et son mandat (l'approche introvertie) ou bien elle peut s'appuyer sur l'environnement politique (l'approche extravertie) ${ }^{33}$.Dans un certain sens, ces approches diffèrent d'une manière fondamentale. Mais ces différentes approches ne s'excluent pas les unes des autres, elles caractérisent aussi les débats qui se déroulent au sein d'une seule organisation. Le personnel sur le terrain, par exemple, pourrait être plus ouvert à l'approche affective alors que les principes traditionnels et juridiques jouent un rôle plus important au siège de l'agence ${ }^{34}$. Vaux souligne que les différentes forces de l'humanitaire qu'il décrit comme «detachment » et «attachment » caractérisent aussi la décision et l'incertitude individuelle ${ }^{35}$.

34 L'approche affective est une réaction émotionnelle plutôt qu'une stratégie délibérée. Dans une telle perspective, il faut se concentrer uniquement sur les personnes affectées par la crise et leurs besoins humanitaires. Cela implique que les facteurs politiques ainsi que les principes de l'action humanitaire soient mis à l'écart, comme l'a dit l'ancien président américain Reagan : "un enfant affamé ne sait rien du politique ». Ce qui compte est la relation entre celui qui fournit l'aide et celui qui la reçoit. L'acte de fournir de l'aide est perçu comme un acte de pitié et de charité plutôt qu'une réponse à un droit à l'assistance du bénéficiaire. L'approche affective nie d'autres obligations que l'impératif humanitaire. Donc, un dilemme n'existe pas. Il faut mettre en exergue que l'approche affective regarde le mandat humanitaire comme un travail apolitique. Le politique et l'humanitaire sont, dans cette approche, des sphères distinctes. Rony Brauman décrit cette conception comme "mystique $»^{36}$. La vulnérabilité des personnes affectées par une crise humanitaire est définie selon les critères purement physiques (âge, sexe, etc.) et prend à peine en compte des facteurs politiques ou sociaux. Les organisations ayant un mandat très large et fondant leur action sur des valeurs autres que l'éthique humanitaire (la religion, la solidarité, la paix, la lutte contre la pauvreté, etc.), sont particulièrement attirées par la démarche affective.

Il faut ajouter qu'une telle démarche peut être très efficace car elle se réfère plus à des images et des anecdotes personnelles qu'aux analyses. Dans la perspective de l'approche affective, la décision de quitter une région en crise pour des raisons éthiques apparaît comme une décision qui ignore l'impératif humanitaire. Prenons encore l'exemple de la Corée du Nord. L'ancien chef de mission de l'ONU commente la décision de quelques ONG de quitter le pays en disant : «Nous ne pouvons pas condamner un enfant coréen à mort seulement parce que cet enfant est né en Corée du Nord $»^{37}$. 
fig. $n^{\circ} 2$ : Les acteurs humanitaires et leurs stratégies face au dilemme

\begin{tabular}{|c|c|c|c|c|}
\hline $\begin{array}{r}\text { Cadre } \\
\text { Ethique }\end{array}$ & 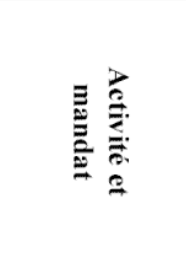 & 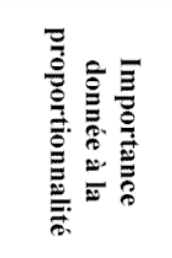 & 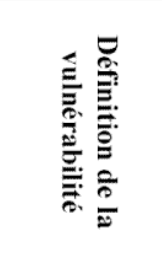 & 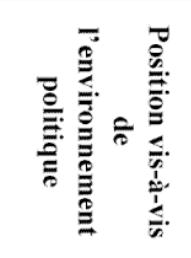 \\
\hline Affective & $\begin{array}{l}\text { réhabilitation, } \\
\text { développement } \\
\text { mandat large } \\
\text { solidarité, } \\
\text { conciliation, } \\
\text { paix etc. }\end{array}$ & $\begin{array}{c}\text { basse } \\
\text { ne fait pas } \\
\text { partie du cadre } \\
\text { éthique }\end{array}$ & $\begin{array}{c}\text { critères } \\
\text { physiques } \\
\text { (age, sexe, } \\
\text { santé, etc.) }\end{array}$ & $\begin{array}{c}\text { apolitique } \\
\text { le politique et } \\
\text { l'humanitarisme } \\
\text { sont des sphères } \\
\text { distinctes }\end{array}$ \\
\hline Introvertie & $\begin{array}{l}\text { aide d'urgence } \\
\text { non médicale, } \\
\text { réhabilitation de } \\
\text { l'agriculture, } \\
\text { développement } \\
\text { mandat large }\end{array}$ & $\begin{array}{c}\text { moyenne } \\
\text { ne fait pas } \\
\text { partie du cadre } \\
\text { éthique }\end{array}$ & $\begin{array}{c}\text { critères } \\
\text { physiques et } \\
\text { économiques }\end{array}$ & $\begin{array}{c}\text { neutre } \\
\text { le politique est } \\
\text { l'environnement } \\
\text { de l'action } \\
\text { humanitaire }\end{array}$ \\
\hline Extravertie & $\begin{array}{l}\text { aide d'urgence } \\
\text { médicale } \\
\text { mandat étroit }\end{array}$ & $\begin{array}{l}\text { haute } \\
\text { fait partie du } \\
\text { cadre éthique }\end{array}$ & $\begin{array}{c}\text { critères } \\
\text { politiques et } \\
\text { sociaux }\end{array}$ & $\begin{array}{c}\text { rebelle } \\
\text { le politique est } \\
\text { l'adversaire de } \\
\text { l'action } \\
\text { humanitaire }\end{array}$ \\
\hline
\end{tabular}

Selon l'approche introvertie, l'acteur humanitaire peut s'orienter vers son propre cadre éthique, ses principes et ses traditions. Ainsi, une organisation d'aide fait référence à ses standards et, le cas échéant, peut les adapter à des nécessités nouvelles. L'organisation elle-même, son mandat et ses principes, sont donc au centre des réflexions. L'adaptation d'une approche commune en matière de principes de travail joue un rôle très important. En Corée du Nord, par exemple, les principes de travail les plus essentiels ont été publiés à plusieurs reprises. Les organisations signataires ellesmêmes considèrent ne pas disposer des moyens nécessaires pour faire respecter ces principes. Néanmoins, l'ONU regarde ces textes comme un succès dans la mesure où les organisations ont trouvé une position commune ${ }^{38}$. Il faut ajouter que ces textes ont été adoptés après que quelques $\mathrm{ONG}$ aient quitté le pays. Ainsi, la référence à des principes communs de l'action humanitaire apparaît comme une réaction au comportement d'autres acteurs au moment d'un débat public plutôt qu'une vraie orientation éthique. Néanmoins, l'approche introvertie n'exclut pas l'option de se retirer d'un pays. Mais la stratégie est d'être patient et d'élargir l'espace de manœuvre à petits pas.

L'argumentation des organisations qui adoptent une stratégie introvertie repose sur une nette distance vis-à-vis des engagements politiques. L'humanitaire est vu comme un travail neutre qui se déroule dans un environnement politique qui, lui, est hors de l'influence des humanitaires. De plus, l'approche introvertie définit les besoins humanitaires d'une population selon des critères physiques et économiques (revenu, emploi, etc). Le cas de la Corée du Nord démontre que les agences de l'ONU et des ONG qui ont plus qu'un mandat médical ont favorisé cette approche.

La troisième approche, l'approche extravertie, se concentre sur l'environnement politique de l'aide, qui est déterminé par des intérêts politiques du côté bénéficiaire et du côté donateur. Selon cette approche, une organisation humanitaire devrait, d'une 
manière active, influencer les décisions politiques si ces dernières ont des effets négatifs sur le travail humanitaire. Autrement dit, dans cette perspective, si c'est le politique qui est à l'origine du dilemme, il faut changer le comportement des acteurs politiques et pas celui des humanitaires. L'approche extravertie n'accepte donc pas les contraintes politiques comme réalité donnée. Selon Alain Destexhe, ancien secrétaire général de MSF International, «l'action humanitaire, depuis et pour toujours, c'est une forme de révolte contre l'inacceptable $»^{39}$. Un des slogans de Médecins du Monde est révélateur à cet égard: "Nous luttons contre toutes les maladies. Même l'injustice». De plus, l'approche extravertie regarde la souveraineté des Etats plutôt comme un obstacle au mandat humanitaire. La branche de l'humanitarisme dit "sans-frontiérisme » a en quelque sorte adopté cette conviction extravertie.

39 La décision de ne pas aider une population est acceptée comme ultime moyen pour exercer de l'influence sur des responsables politiques. Selon Rony Brauman, ancien président de MSF France, il faut "savoir dire non » ${ }^{40}$. L'approche extravertie n'hésite donc pas à prendre des positions nettement politiques, tout en tenant la distance vis-àvis des acteurs politiques. Par rapport à la définition de la vulnérabilité, il est évident que les critères politiques, notamment la distribution du pouvoir politique au sein d'une société, jouent un rôle décisif. Le cas de la Corée du Nord illustre qu'une telle analyse politique peut être douteuse. Ce sont surtout les organisations de l'aide médicale d'urgence qui adoptent une stratégie extravertie. Selon le mandat d'un médecin, la proportionnalité de l'aide détermine si une mission est un succès ou un échec.

40 Les différentes approches des acteurs humanitaires par rapport aux conflits éthiques, y compris les situations de dilemme, révèlent que les organisations humanitaires n'ont pas de positions communes en ce qui concerne les principes les plus fondamentaux de leur travail ${ }^{41}$. Il n'est ni désirable, ni possible de créer une communauté homogène d'acteurs humanitaires. Or, les cas de l'histoire récente de l'humanitarisme, comme celui de la Corée du Nord, montrent que la diversité des acteurs est devenue hautement problématique. Il dépend du cadre éthique de chaque organisation individuelle de déterminer le contenu et l'importance des principes de l'impartialité et de la proportionnalité. Dès lors, ces principes sont devenus flous. C'est cette confusion qui est à l'origine de la crise actuelle du mouvement humanitaire qui est donc, en premier lieu, une crise de cohérence.

$41 \mathrm{Au}$ lieu d'introduire une nouvelle éthique humanitaire, c'est le dialogue entre les organisations d'aide qui pourrait définir une base morale commune de l'action humanitaire. L'objectif devrait être une nouvelle balance entre l'impératif humanitaire, les traditions des organisations individuelles et les réalités politiques. Bien évidemment, cela n'éradiquera pas toute ambiguïté et tout dilemme. Tant que l'action humanitaire cherchera à soulager la souffrance des hommes, des conflits éthiques seront inévitables. L'objectif serait donc de réduire l'incohérence de l'aide humanitaire d'aujourd'hui et de rétablir sa crédibilité. 


\section{NOTES}

1. Vaux T., The Selfish Altruist. Relief Work in Famine and War, Londres, Earthscan, 2001, p. 43.

2. . Fox F., «New humanitarianism. Does it provide a moral banner for the 21st century? ", Disasters, vol.25, $\mathrm{n}^{\circ} 4,2001$, p. 288 . Notre traduction. Les traductions suivantes sont également de l'auteur.

3. . Lindenberg M, Bryant C., Going Global. Transforming relief and development NGOs, Bloomfield Kumarian, 2001, p. 76.

4. . Lindenberg M, Bryant, C., ibid., p. 76-77.

5. . Leader N., The Politics of Principle. The Principles of Humanitarian Action in Practice, London, Overseas Development Institute, 2000, p. 6.

6. . Ibid.

7. James Orbinski, ancien président de MSF International, discours à Oslo, 10 décembre 1999, www.doctorswithoutborders.org/publications/speeches/1999/jo_nobel.shtml (21 juillet 2003).

8. . Brauman R., Humanitaire : le dilemme, Paris, Editions Textuel, 1996, p. 39.

9. . Fox F., «New humanitarianism. Does it provide a moral banner for the $21^{\text {st }}$ century? ", Disasters, vol. 25, n4, 2001, p. 275.

10. . Voir par exemple "Code of Conduct for Humanitarian Agencies in Sierra Leone", "Principles of Engagement for Humanitarian Assistance in the Democratic Republic of Congo ", "Joint Policy of Operation in Liberia ", " Ground Rules Agreement in south Sudan », "Statement of Humanitarian Principles - Democratic People's Republic of Korea ».

11. . Leader N., "Proliferating principles. Or how to sup with the devil without getting eaten ", Disasters, vol.22, n4, 1998, p. 290.

12. . Active Learning Network for Accountability and Performance in Humanitarian Action (ALNAP), Humanitarian action. Learning from evaluation, Londres, ALNAP, 2001, paragraphe 4.2.5.

13. Schloms M., North Korea and the Timeless Dilemma of Aid. A Study of Humanitarian Action in Famines, Berlin, Londres, Lit Verlag, 2004, pp. 268-271.

14. . Boltanski L., Distant suffering. Morality, Media and Politics, Cambridge, Cambridge University Press, 1999, p. 191.

15. . Slim H., « Not philanthropy but rights, Rights-based humanitarianism and the proper politicisation of humanitarian philosophy in war », présentation lors de la conférence Politics and Humanitarian Aid. Debates, Dilemmas and Dissension, Londres, $1^{\text {er }}$ février 2001, p. 20.

16. . Ibid.

17. . Anderson M., Do no harm. How Aid can support Peace - or War, Londres, Lynne Rienner, 1999, p. 146.

18. . Destexhe A., L'Humanitaire impossible ou deux siècles d'ambiguïté, Paris, Armand Colin, 1993, p. 7.

19. . Sinnott-Armstrong W., Moral Dilemmas, Oxford, Basil Blackwell, 1998, p. 29.

20. . Slim H., « Doing the Right Thing. Relief Agencies, Moral Dilemmas and Moral Responsibility in Political Emergencies and War », Disasters, vol.21, n³, 1997, p. 249.

21. . Hermet G., "Triomphe ou déclin de l'humanitaire? », in Cultures et Conflits, n¹1, 1993, p. 15.

22. . Pour la genèse des famines, le rôle des facteurs politiques et les implications pour l'action humanitaire voir Schloms M., North Korea and the Timeless Dilemma of Aid. A Study of Humanitarian Action in Famines, op. cit., pp. 30-51

23. . Ibid, pp. 112-120 
24. . Le Programme Alimentaire Mondial, quant à lui, a mobilisé plus d'1,2 milliard de US dollars avant la fin de l'année 2003 pour les opérations en Corée du Nord.

25. . Schloms M., North Korea and the Timeless Dilemma of Aid. A Study of Humanitarian Action in Famines, op. cit., pp. 156-215.

26. . Il y en a d'autres qui se sont retirées de la Corée du Nord pour des raisons diverses. L'ONG allemande Cap Anamur a pris cette décision en disant que les restrictions en place rendaient les efforts trop lents et trop inefficaces. L'ONG américaine CARE, quant à elle, a cessé ses activité dans le pays pour des raisons plutôt internes. Voir Flake L.G., « The Experience of U.S. NGOs in North Korea ", in Flake L.G., Snyder, S., Paved with Good Intentions. NGO Experience in North Korea, Westport, Praeger, 2003, pp. 30-31.

27. Communiqué de presse de MSF, Paris, 30 septembre 1998.

28. . Dossier de presse d'ACF «Action Contre La Faim Décide de se retirer de la Corée du Nord », Paris, mars 2000, p. 1.

29. . Ibid., p. 9.

30. . Voir Schloms M., North Korea and the timeless dilemma of aid. A study of humanitarian action in famines, op. cit., pp. 106-112.

31. . Vaux T., The Selfish Altruist. Relief Work in Famine and War, Londres, Earthscan, 2001, pp. 123-127.

32. . Schloms M., North Korea and the Timeless Dilemma of Aid. A Study of Humanitarian Action in Famines, op. cit., pp. 277-281.

33. . Voir la figure $n^{\circ} 2:$ « Les acteurs humanitaires et leur stratégie face au dilemme ».

34. . Pour une analyse des débats qui opposent des différentes sections d'une organisation internationale, voir Dauvin P., « Kosovo : histoire d'une déportation ou la chronique d'une prise de parole publique dans une ONG internationale ", in Dauvin P., Siméant J., ONG et humanitaire entre militantisme transnational et action publique, Paris, L'Harmattan, 2004, pp. 35-39.

35. . Vaux T., The Selfish Altruist. Relief Work in Famine and War, op. cit.

36. . Brauman R., Humanitaire : le dilemme, op. cit., p. 39 : «En ce qui me concerne, je réfute toute conception mystique de l'humanitaire. L'humanitaire fait partie de ces actions où une forme dure de réalisme est particulièrement importante, justement parce qu'on est dans le royaume de l'effusion, du sentiment et des passions, et que ce terrain est très vite glissant. Il faut au contraire avoir les pieds sur terre, un minimum de réflexion politique et de capacités de discernement, pas la larme à l'œil ni le chapelet à la main ».

37. Schloms M., North Korea and the Timeless Dilemma of Aid. A Study of Humanitarian Action in Famines, op. cit., p. 219

38. Schloms M., North Korea and the Timeless Dilemma of Aid. A Study of Humanitarian Action in Famines, Berlin, London, Lit Verlag, op. cit., pp. 223-224.

39. . Destexhe A., L'Humanitaire impossible ou deux siècles d'ambiguïté, Paris, Armand Colin, 1993, p. 216.

40. . Brauman R., L'Action humanitaire, Paris, Flammarion, 1995, p. 99.

41. Voir figure $n^{\circ} 2$. 


\section{RÉSUMÉS}

Cet article analyse le débat actuel sur le dilemme humanitaire et la crise de l'action humanitaire. Il met en question la diagnose et les conclusions dominantes. L'article présente l'ambiguïté comme un phénomène structurel de l'action humanitaire. L'analyse des cas concrets du dilemme humanitaire, notamment de la Corée du Nord, révèle différentes catégories d'organisations humanitaires. L'auteur conclut que l'humanitarisme est caractérisé par un état de confusion par rapport aux principes les plus fondamentaux de l'action humanitaire. Ainsi, les organisations d'aide font face à une crise de cohérence.

This article analyses the contemporary debate about the humanitarian dilemma and the crisis of humanitarian action. The author challenges the diagnosis and the dominant conclusions that are usually offered. The article shows that the ambiguity is a structural phenomenon of humanitarian action. The empirical case studies, primarily about North Korea, reveal different types of humanitarian organisations. The author concludes that humanitarianism is characterised by a state of confusion concerning the role of the most fundamental principles of humanitarian action. As a consequence, the humanitarian relief agencies are faced with a crisis of coherence.

\section{INDEX}

Mots-clés : humanitaire

\section{AUTEUR}

\section{MICHAEL SCHLOMS}

Michael SCHLOMS est diplômé de l'Université Libre de Berlin et de l'Institut d'Etudes Politiques de Paris. Il a soutenu sa thèse en 2003 sur l'éthique humanitaire et le cas de la Corée du Nord. Celle-ci a été publiée en 2004 sous le titre North Korea and the Timeless Dilemma of Aid. A Study of Humanitarian Action in Famines. 